\title{
HIGH-PRECISION SILICON MICROMACHINED MICROMIRRORS FOR LASER BEAM SCANNING AND POSITIONING
}

\author{
Meng-Hsiung Kiang, Olav Solgaard*, Richard S. Muller and Kam Y. Lau \\ Department of Electrical Engineering and Computer Sciences \\ University of Califomia at Berkeley \\ Berkeley, CA 94720 \\ *Department of Electrical and Computer Engineering \\ University of Califomia at Davis \\ Davis, CA 95616
}

\begin{abstract}
We present the design and fabrication of surfacemicromachined micromirrors actuated by electrostatic-comb motors. High-precision positioning over a large angular range makes the micromirrors suitable for a variety of optical applications such as laser scanners and printers, displays, holographic-data storage, and fiber- optic switches.
\end{abstract}

\section{INTRODUCTION}

Silicon-surface-micromachining technology has been used to fabricate micro-optical devices such as movable-micromirrors for building optical components and communication subsystems [1-4]. Motorized micromirrors with actuators integrated on the same chip (such as those in [1-2]) are of particular interest because they make possible truly miniaturized systems. For practical optical systems, the smallest optical aperture (such as that of the mirror in our case) has to be large enough to insure sufficient image resolution. Polysilicon microhinges, which allow the micromirrors to be lifted out of the plane of the substrate after processing is completed, are used to create high-aspect-ratio optical surfaces with dimensions in the hundreds of $\mu \mathrm{ms}$ while taking advantage of the planar surface-micromachining processing technology. Polysilicon torsion bars are used for mirror suspension in these structures and also for as the rotation shafts for the scanner.

We present here the design and fabrication of siliconsurface-micromachined scanning micromirrors that have high angular precision over a large scan angle. When used as resonant scanners, these mirrors have fast scan rates with very low operating power. We will discuss single-mirror scanners that are capable of high-speed scanning with a large scan angle. These single-mirror scanners can be combined to form more complicated microscanners such as a two-mirror, two-axis raster scanner that can have a wide range of applications in areas such as communications, medicine, and entertainment, among others.

\section{ACTUATED MICROMIRROR DESIGN}

The scanning micromirrors are fabricated using siliconsurface-micromachining processes [1,2]. Figure 1 shows SEM photographs of two comb-actuated micromirrors having orthogonal axes of rotation. The electrostatic comb drive for this scanner system consists of 100 interdigitated fingers for both the shuttle and the stationary comb on the two sides, with lateral dimensions totaling $1 \mathrm{~mm}$. The motor is attached through a pair of restoring springs to a base plate that is hinged to the bottom of the micromirror. The double-folded restoring springs with truss suspensions have beams $2 \mu \mathrm{m}$ wide and $300 \mu \mathrm{m}$ long. The mirrors are mounted on the frames through two torsion bars (each $2 \mu \mathrm{m}$ (a)

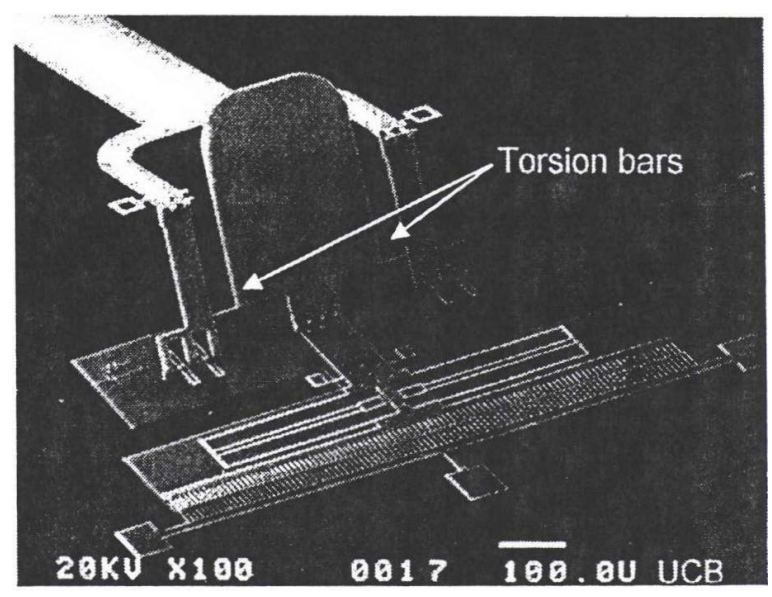

(b)

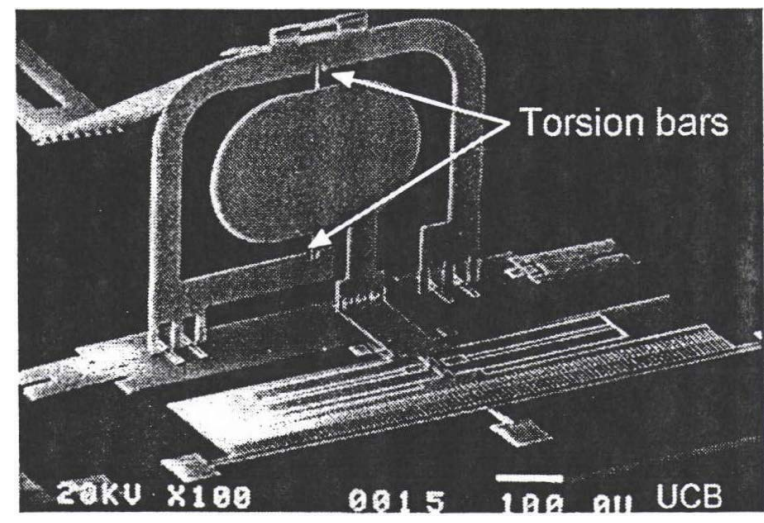

Fig. 1 SEM photographs of two electrostatic-comb-driven micromirrors having two different axes of rotation to enable scanning along two orthogonal axes. Both micromirrors measure 300 by $500 \mu \mathrm{m}$. a: $\theta$ (vertical)-scan mirror; $\boldsymbol{b}: \phi$ (lateral)-scan mirror.

wide and $50 \mu \mathrm{m}$ long ) as the pivoting shafts for the mirrors. Two layers of structural polysilicon (each $2-\mu \mathrm{m}$ thick) are used to complete the scanner structure. LPCVD silicon dioxide is used as the sacrificial material separating the different polysilicon layers.

\section{CHARACTERIZATION}

To characterize the - performance of the scanning micromirrors, a position-sensing photodetector is used to record the position of a He-Ne laser beam reflected off the surface of the actuated micromirrors. The mirror in Fig. 1a is used first in our measurements. With varying dc-bias voltage (up- and down-ramp over many voltage cycles) to the electrostatic comb drive, the corresponding position of the reflected laser beam is plotted in 
Fig. 2. The measurements show an angular position accuracy of $0.662 \mathrm{mrad}$ in standard deviation. Over a total scan range of 0.1 rad, corresponds to 150 resolvable static-positions. On the other hand, if instead we drive the comb motor with an ac voltage close to or at its resonant frequency, the scanning laser beam scans in a smooth sinusoidal fashion over an excursion angle of $10^{\circ}$. It deviates from the sine-wave motion by $9.6 \mu \mathrm{m}$, or $0.19 \mathrm{mrad}$, in standard deviation along the scan line. This is a measure of the scan-line repeatability which is an-important issue for optical scanning applications. Figure 3 is a measured result from the $\phi-$ scan mirror in Fig. 1b, showing also the sinusoidal motion over an excursion angle of $7^{\circ}$.

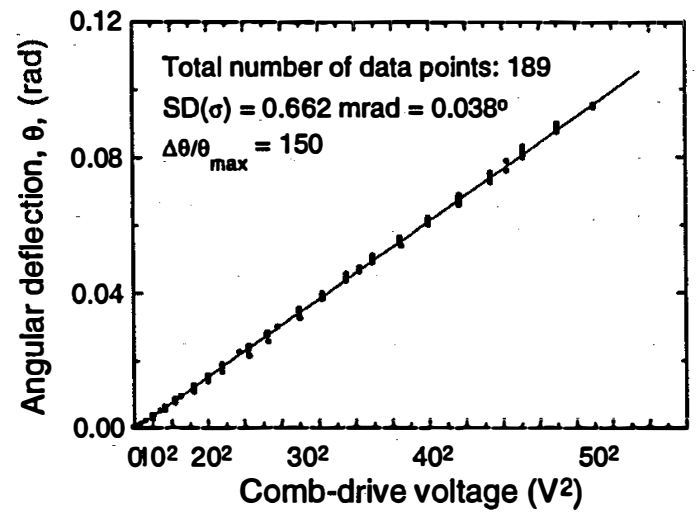

Fig. 2 The static response of the $\theta$-scanning micromirror to varying dc-bias voltage.

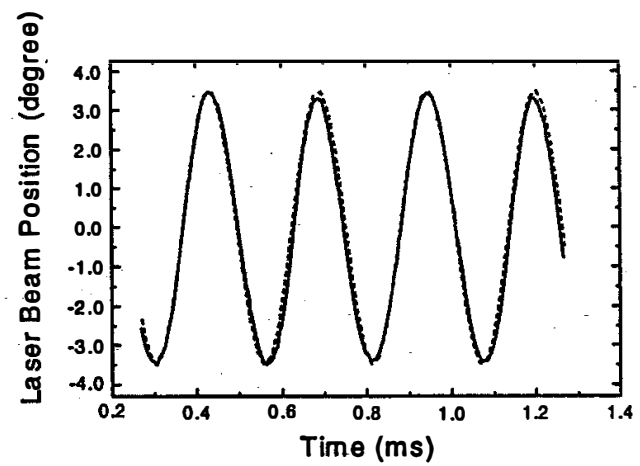

Fig. 3 Scanning laser beam (deflected by the mircroscanner shown in Fig. 1b) recorded with a position-sensing detector. The motor of the microscanner is driven by an ac voltage $30 \mathrm{~V}$ in amplitude at $2 \mathrm{kHz}$.

\section{DISCUSSION}

The use of torsion bar suspension provides accuracy in the control of the rotation angles. If we perform the same measurement as that for Fig. 2 with another scanning micromirror from our previous design [2] which uses micropin-hinges as the mirror shaft, the angular precision obtained (plotted in Fig. 4) is $3.3 \mathrm{mrad}$ (compared with $0.662 \mathrm{mrad}$ in Fig. 2). With a total scan angle of $0.21 \mathrm{rad}$, this corresponds to 63 resolvable static positions over the scan range. This reduction in angular accuracy can be attributed to the difference in the rotation-shaft structures for the two designs. As shown in the inset in Fig. 4, the armature of the micropin-hinge turns against a square shaft when the mirror rotates. As a result, the friction and drag cause a larger angular inaccuracy in mirror position. In addition, the wear in the shaft structure in the micropin-hinge could potential be a concern for long-term reliability of the system.

The maximal scan angles in our present design, on the other hand; have been reduced from our previous case [2]. This is the result of using only two layers of polysilicon in the present structure that does not allow a mechanical design to compensate for the change in the elevation or the angle of the mirror base as the mirror rotates. Double-hinged base plate structure made of three layers of structural polysilicon (as that in [2]) will alleviate the problem and enable $\mathrm{us}$ to increase the scan range further.

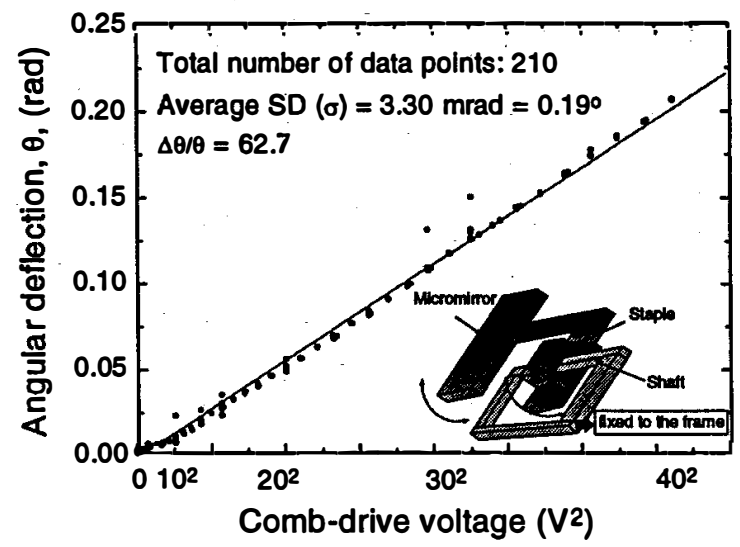

Fig. 4 The static responses of a $\theta$-scan micromirror (described in [2]) to varying dc bias-voltage. The microscanner is a threepolysilicon structure with micropin-hinges as the rotation shafts.

\section{CONCLUSION}

We have designed and fabricated high precision polysilicon microscanners using surface micromachining. Electrostatic-comb actuators drive 300 by $500 \mu \mathrm{m}$ micromirrors mounted on torsion bars. The microscanners are accurate to $0.662 \mathrm{mrad}$ over $0.1 \mathrm{rad}$ total scan range. In terms of static positioning, this corresponds to 150 resolvable positions, an almost three-fold improvement from structures using micropin-hinge design. This type of microscanner has previously been demonstrated as the scanner for a barcode reader [2]: Now, with higher angular precision, these electrostatic-comb-actuated micromirrors are also suitable for laser beam steering in more demanding optical scanning applications.

\section{REFERENCES}

[1] M.-H. Kiang, O. Solgaard, R. S. Muller, and K. Y. Iau, "Silicon-Micromachined Micromirrors with Integrated HighPrecision Actuators for External Cavity Semiconductor Lasers," IEEE Photon. Technol. Lett., Vol. 8, no. 1, pp. 9597, 1996.

[2] M.-H. Kiang, O. Solgaard, R. S. Muller, and K. Y. Lau, "Surface-Micromachined Electrostatic-Comb Driven Scanning Micromirrors for Barcode Scanners," Proceed. IEEE Micro Electro Mechanical Systems, San Diego, CA, 2/12-16/96, pp. 175-180.

[3] O. Solgaard, M. Daneman, N. C. Tien, A. Friedberger, R. S. Muller, and K. Y. Lau, "Optoelectronic Packaging Using Silicon Surface-Micromachined Alignment Mirrors,". IEEE Photon. Technol. Lett., Vol. 7, no. 1, pp. 41-43, 1995.

[4] - Y. Lin; S. S. Lee, K. S. Pister, and M. C. Wu, "Micromachined Three-dimensional Micro-optics for Integrated Free-space Optical Systems," IEEE Photon. Technol. Lett., Vol. 6, no. 12, pp. 1445-1447, 1994. 